\title{
Behavior of Sandwich Structures and Spaced Plates Subjected to High-Velocity Impacts
}

\author{
Shirley K. García-Castillo, ${ }^{1}$ Brenda L. Buitrago, ${ }^{1,2}$ Enrique Barbero ${ }^{1}$ \\ ${ }^{1}$ Department of Continuum Mechanics and Structural Analysis, University Carlos III of Madrid, \\ Avda. de la Universidad 30, 28911 Leganés, Madrid, Spain \\ ${ }^{2}$ Department of Industrial Technology, University Simón Bolivar, Valle de Sartenejas, Caracas, Venezuela
}

\begin{abstract}
This work evaluates the behavior of sandwich and spaced plates subjected to high-velocity impacts. The sandwich structures were made of glass/polyester face-sheet and a PVC foam core. The spaced plates were made of two plates of the same material of the sandwich face-sheet at a distance equal to the core thickness. The residual velocity, the ballistic limit, and the damage area were selected to compare the response of both structures. The residual velocity and ballistic limit was very similar in both cases. Nevertheless, the damage area of sandwich structures and spaced plates differed due to the dissimilar properties between the sandwich core and the air inside of the spaced plates. An analytical model, based on energy criteria, was applied to estimate the residual velocity of the projectile, the absorbed energy by each facesheet, and the ballistic limit in the spaced plates. POLYM. COMPOS., 00:000-000, 2010. ( 2010 Society of Plastics Engineers
\end{abstract}

Keywords: high velocity impact; sandwich; foam; damage

\section{INTRODUCTION}

Composite structures offer a variety of potential advantages over metal structures, such as light weight, stiffness, and strength. Nevertheless, theses structures have low transverse stiffness, which could cause failure under concentrated loads [1, 2]. During their service life, these kinds of structures could be subjected to high-velocity impacts of low-mass fragments, the effects ranging from indentation to complete perforation of the structure. Such damage constitutes an important risk for such structures because, although they are not designed as armor, the mechanical properties after impact are reduced. The mechan-

Correspondence to: Dr. Shirley K. García Castillo; e mail: sgcastil@ing. uc3m.es

Contract grant sponsor: Spanish Comisión Interministerial de Ciencia y Tecnología; contract grant number: TRA2007 66555. ical properties of a composite material are affected by the impact velocity [3] and therefore it is necessary study the impact behavior of composite structures $[4,5]$.

Some authors suggest that the specific energy-absorption capability of thin laminates subjected to high velocity surpasses than that of thick laminates [6]. This inspired the use of multiple thin laminate plates to protect against a high-velocity impact, although there are some discrepancies about whether a layered composite structure with or without spacing is better than a monolithic one [7].

Another type of structure widely employed is the sandwich because of their high flexural strength and stiffness, which additionally could offer energy-absorbing capabilities for crashworthiness [1, 8, 9]. Low-velocity impact behavior of sandwich structures has been the focus of many studies [1, 10], whereas fewer studies examine the behavior of sandwich structures subjected to high-velocity impact [11]. The analysis of sandwich structures subjected to high-velocity impacts is more complex than the metal structures, due to complex interaction of the face-sheet and core [12].

One parameter that can be used to estimate the strength of a structure to perforation is the ballistic limit. This can be defined as the maximum velocity at which a particular projectile is expected to consistently fail to penetrate the component [13]. This velocity can be determined by means of experimental tests or by theoretical models. In sandwich structures the estimation of the ballistic limit by theoretical models is complex due to the interaction of the face-sheet and core [9].

The residual strength after impact of a composite structure is related to the damage area. Even when the damage is not visible in the structure the mechanical properties could be reduced [14]. Furthermore, it has been observed that in high-velocity impacts the damage generated close to the ballistic limit can be extensive, and therefore, in the design of the structure, it is important to know this damage [15]. There are several damage mechanisms in a laminate, including matrix cracking, fiber failure, and 


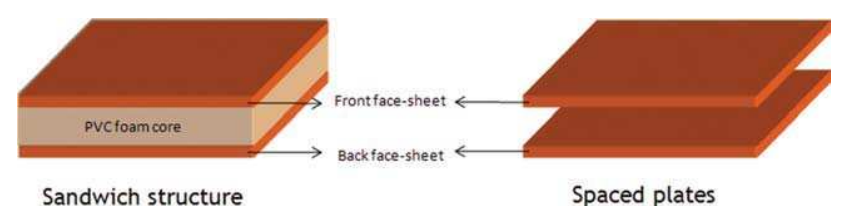

FIG. 1. Specimens.

delamination. In a sandwich structure these mechanisms are combined with the debonding between the face-sheet and core. The damage generated by the impact could be understood by the knowledge of the absorbed energy in the structure [16].

This study examines by experimental tests the behavior of sandwich structures and spaced plates when they are subjected to impacts of low-mass projectiles. The residual velocity, ballistic limit and damage area were evaluated in both structures.

In the spaced plates, an analytical model was used to evaluate the residual velocity of the projectile after the perforation and to estimate the energy absorbed by the front and back face-sheet. The impact process was studied by modeling the face-sheets, using an analytical model developed for laminates and applying these models to each face-sheet separately. There are many models to evaluate the impact behavior of laminates, some based on energetic criteria $\left[\begin{array}{ll}17 & 20]\end{array}\right]$. In this work the model proposed by García-Castillo et al. [20] was used. This model was validated experimentally and numerically for woven laminates and it enables the determination of the residual velocity of the projectile, the ballistic limit, and the contributions of several mechanisms to the absorption of the kinetic energy of the projectile.

\section{EXPERIMENTAL PROCEDURES}

The face-sheets of the structures used in this study were made from glass-E/polyester woven laminates with a thickness of $3 \mathrm{~mm}$. The distance between the facesheets in both structures was the same as the core thickness, $30 \mathrm{~mm}$. The core of the sandwich was a PVC foam with a density of $100 \mathrm{~kg} / \mathrm{m}^{3}$ (Fig. 1). The dimensions of the specimens were $160 \mathrm{~mm} \times 160 \mathrm{~mm}$. This size guaranteed that the damage would not reach the edge of the specimen and therefore the boundary conditions did not influence the damage [21].

A gas gun, Sabre Ballistics model A1G +, was used in the impact tests. Impact velocity was controlled by regulating the pressure in the system. In a previous study [22], an experimental correlation between pressure and velocity of the projectile was estimated. The correlation was used to select the pressure to achieve the required impact velocity, from 80 to $772 \mathrm{~m} / \mathrm{s}$. Spherical steel projectiles of $7.5 \mathrm{~mm}$ in diameter and a mass of $1.7 \mathrm{~g}$ were used.

A high-speed video PHOTRON FASTCAM-ultima APX was used to record the impact tests. The data-acquisition system of the camera was adjusted to gather information in a window of 50,000 frames per second. A good resolution of the projectile path was achieved, as shown in Figure 2, both in the front face-sheet (Fig. 2a), and in the back face-sheet (Fig. 2b).

From the information provided by the camera, the impact and residual velocity was calculated, evaluating the distance traveled by the projectile in several consecutive frames. The number of frames was selected according to a previous study to ensure an accurate estimation of the velocity [22].

The damage area was calculated using digital imageprocessing software from photographs of the impacted specimens; this was possible because the face-sheets were of a translucent material [23]. Figure 3 shows the evaluation process of one of the impacted specimens. Figure 3a displays the original image, the contour of the damage is marked by the software in Figure $3 \mathrm{~b}$, and the area inside this contour is calculated in Figure 3c.

\section{Experimental Results}

The residual velocity of the projectile after perforation is shown in Figure 4, for both the sandwich structure and spaced plate. Lambert and Jonas [24] affirm that the residual velocity of the projectile $\left(v_{\mathrm{R}}\right)$ is correlated with the impact velocity $\left(v_{0}\right)$ by Eq. 1:

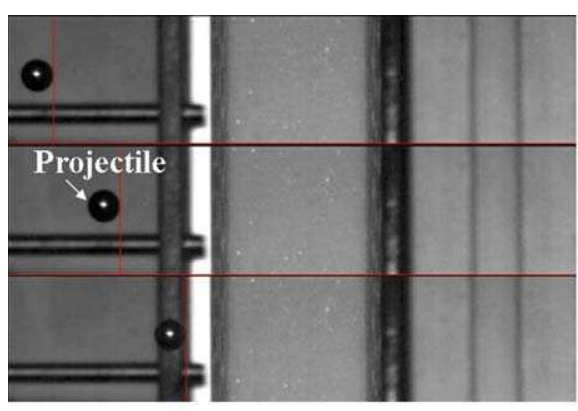

(a)

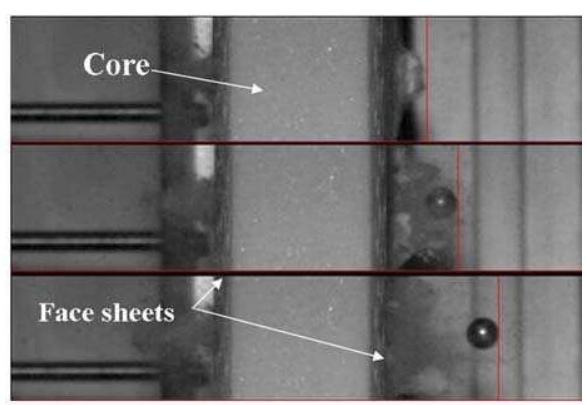

(b)

FIG. 2. Displacement of the projectile (a) before perforation and (b) after perforation. 


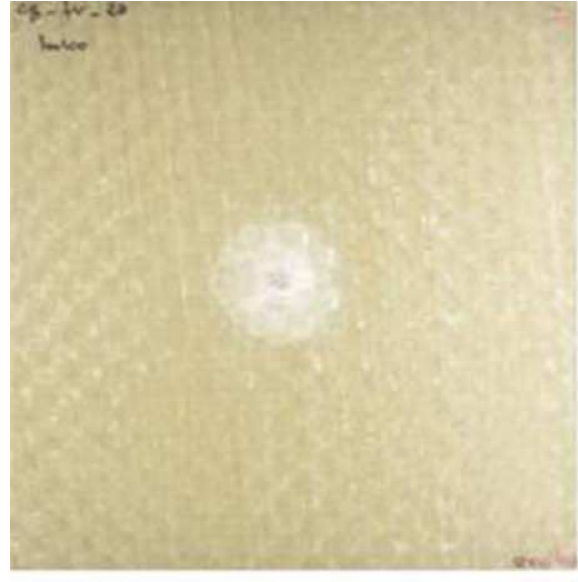

(a)

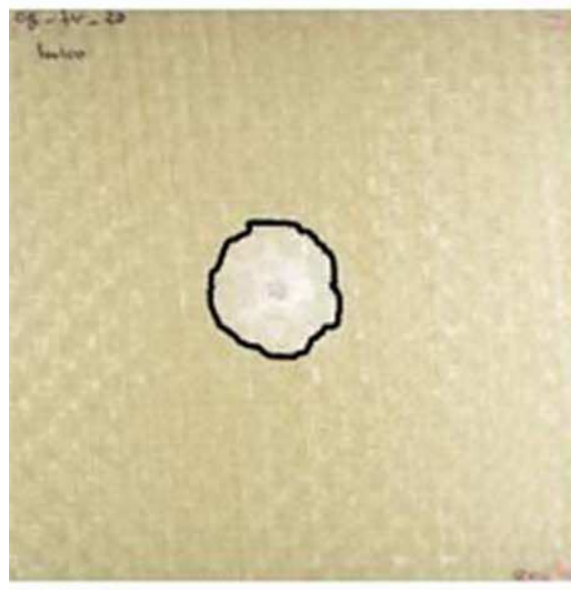

(b)

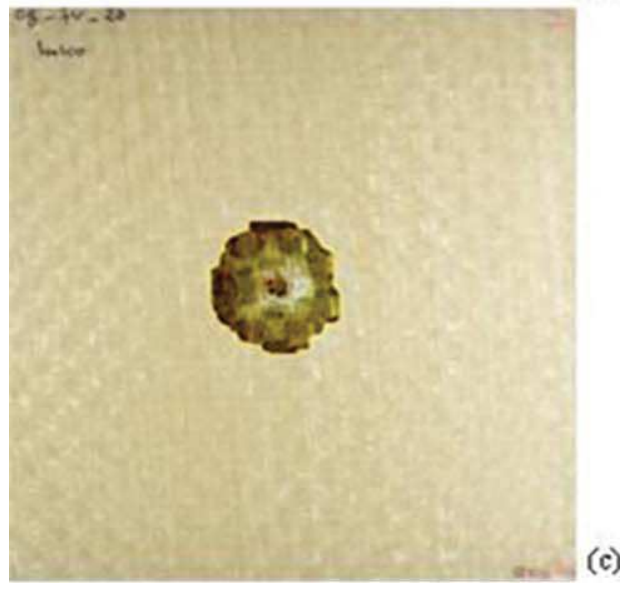

FIG. 3. Damage area of the front face sheet of a sandwich plate impacted at $657 \mathrm{~m} / \mathrm{s}$. (a) Original image. (b) Contour determination. (c) Area evaluation.

$$
v_{\mathrm{R}}= \begin{cases}0 & 0<v_{0} \leq v_{\mathrm{BL}} \\ A \cdot\left(v_{0}^{p}-v_{\mathrm{BL}}^{p}\right)^{1 / p}, & v_{0}>v_{\mathrm{BL}}\end{cases}
$$

where, $v_{\mathrm{BL}}$ is the ballistic limit, $A$ and $p$ are empirical parameters. In this work the values of the parameter were 0.9 and 2, respectively.

Because of the difficulty of controlling impact velocities precisely and the existence of a zone of mixed results in which a projectile may completely perforate or

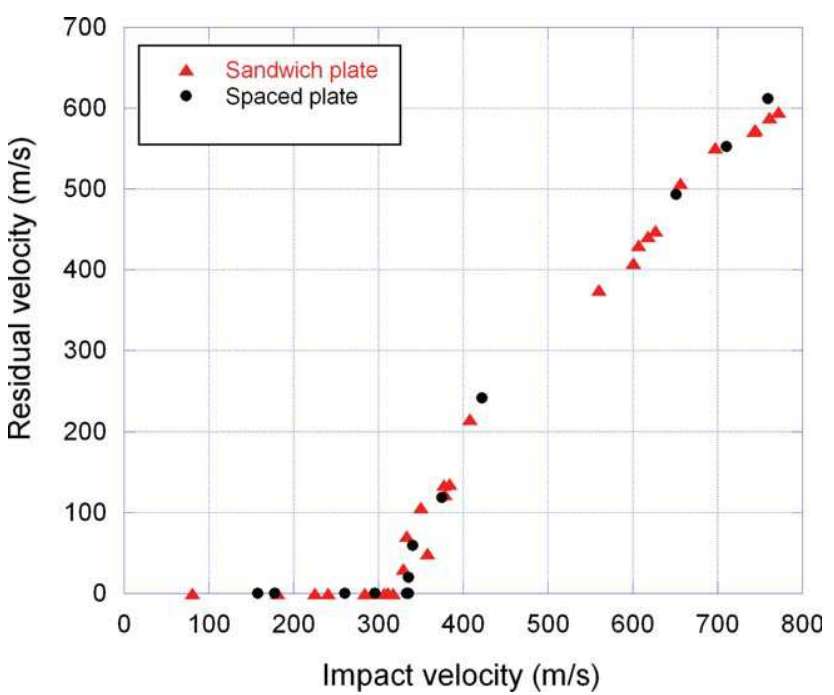

FIG. 4. Residual velocity vs. impact velocity.

only partially penetrate under apparently identical conditions, the ballistic limit cannot be calculated in a deterministic way [13]. In this work the Eq. $I$ was fit to the experimental data of Figure 3 by the least-squares method. Table 1 shows the ballistic limit estimated for the sandwich plate and spaced plate by this equation. The difference between the two values is $2.6 \%$. These results indicate that the core has no significant influence on the ballistic limits.

Damage area in the face-sheets of both structures (Figs. 5 and 6) showed the same trend as that found in laminates of the same materials [21]. The damage increased significantly with the impact velocity below the ballistic limit and decreased above the ballistic limit.

The damage area in the front face-sheet was greater in the spaced plate than in the sandwich plate (Fig. 7). On the contrary, in the back face-sheet the greatest damage was found in the sandwich structure (Fig. 8). This result shows that the core influences the damage area of the sandwich plate.

There are significant differences in the damage in the front face-sheet, especially for impact velocities close to the ballistic limit, where the damage area of the spaced plate was $40 \%$ larger than the damage area on the front face-sheet sandwich structure. In the back face-sheet the behavior was the opposite, the damage area being $60 \%$ smaller in the spaced plate.

TABLE 1. Experimental ballistic limit of structures of composite materials.

\begin{tabular}{lcc}
\hline Structures & Sandwich structure & Spaced plate \\
\hline Ballistic limit $(\mathrm{m} / \mathrm{s})$ & 344 & 335 \\
\hline
\end{tabular}




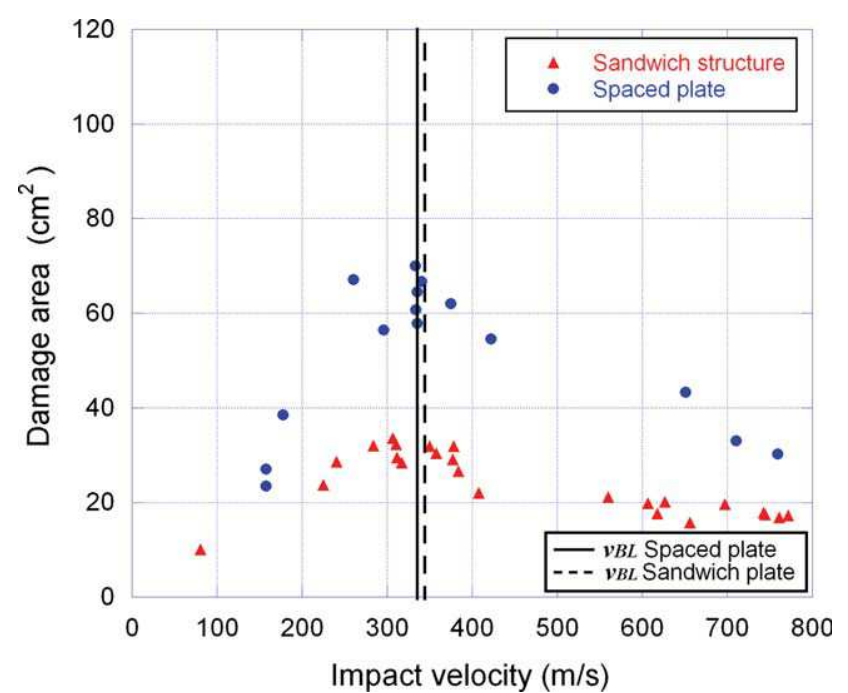

FIG. 5. Damage area vs. impact velocity in the front face sheet.

The differences in the damage area in the front and back face-sheet of the structures studied could be influenced by the propagation of elastic waves, which is controlled by the differences in the properties (density and young modulus) among the glass-polyester face-sheets, PVC foam core, and air. In the sandwich structure, part of the wave generated by the impact is reflected in the face-sheet/core interface and another smaller part is transmitted to the core, while in the spaced-plate structure the wave is not transmitted to the back-face sheet due to the absence of a material between both face-sheets, making the damage area in the front face-sheet larger in this structure. By contrast, the damage area in the back facesheet of the sandwich structure was larger because the

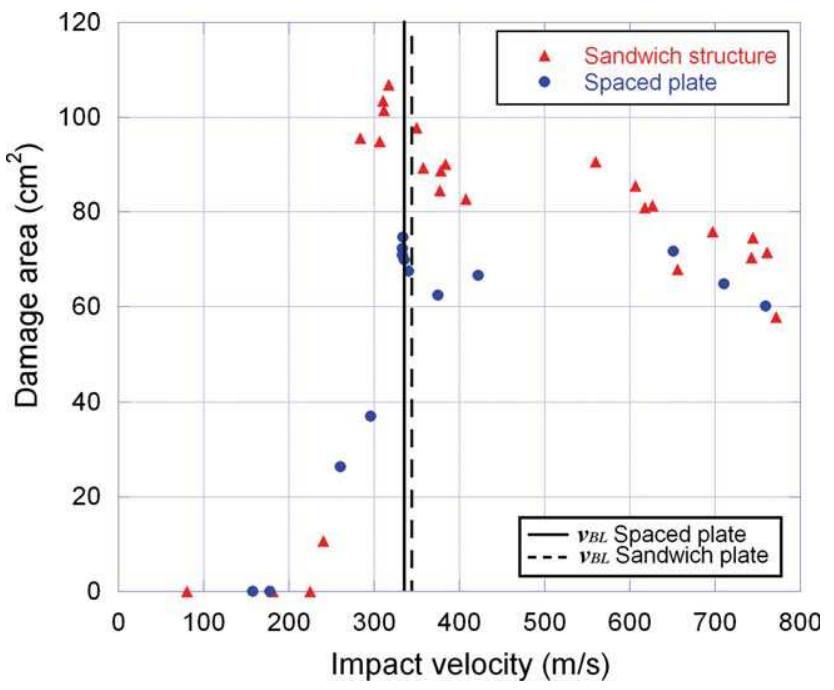

FIG. 6. Damage area vs. impact velocity in the back face sheet.

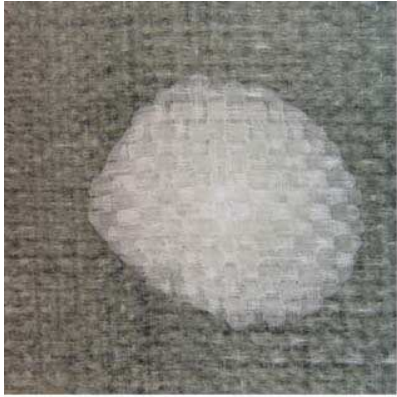

(a)

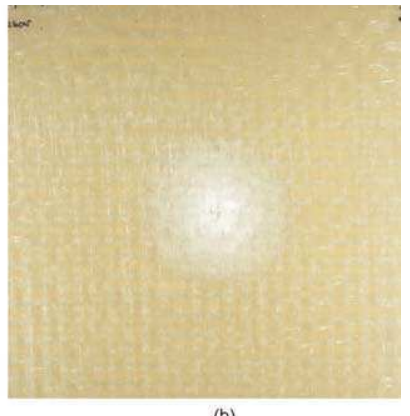

FIG. 7. Image of damage area on front face sheet. (a) spaced plate. (b) sandwich plate.

transmitted wave is amplified due to the far greater rigidity of the glass-polyester than of the PCV-foam.

\section{Modeling of Spaced Plates}

In this work the analytical model proposed by GarcíaCastillo et al. [20] was applied to the spaced plate. The model was developed for woven laminates and is based on energy criteria. It allows the estimation of the residual velocity of the projectile, the ballistic limit, the energy absorbed by different mechanisms during the penetration of the laminate and the contact time between the projectile and the plate. This model was validated with experimental tests and numerical simulations for glass/polyester laminate.

The model considers three energy-absorption mechanisms: the kinetic energy of the moving cone formed on the back side of the plate $\left(E_{\mathrm{KC}}\right)$, the energy absorbed by elastic deformation of secondary yarns $\left(E_{\mathrm{ED}}\right)$, and the energy absorbed by the failure of the plate $\left(E_{\mathrm{FP}}\right)$, this latter mechanism included: the failure of primary yarns $\left(E_{\mathrm{TF}}\right)$, damage by delamination $\left(E_{\mathrm{DL}}\right)$, and matrix cracking $\left(E_{\mathrm{MC}}\right)$. These energy-absorption mechanisms are detailed in Ref. 20.

In the analytical model the primary yarns are the fibers directly below the projectile, which offers resistive force
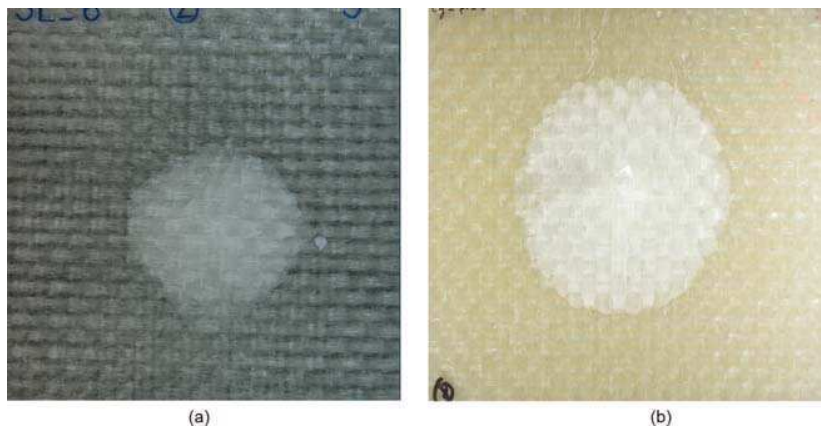

FIG. 8. Image of damage area on back face sheet. (a) Spaced plate. (b) Sandwich plate. 


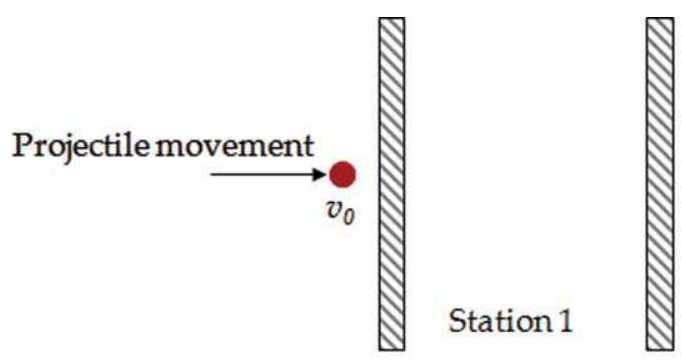

(1)

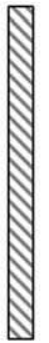

(2)

$\mathbb{N}$

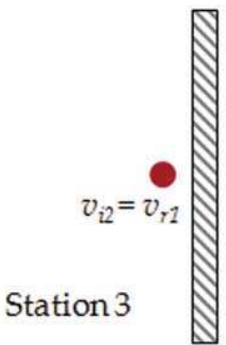

Front face-sheet (1)

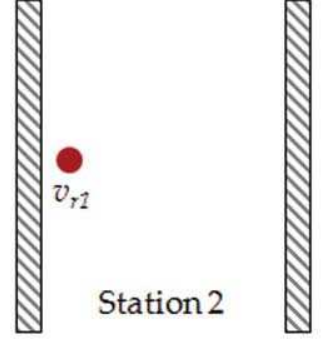

(1)

(2)

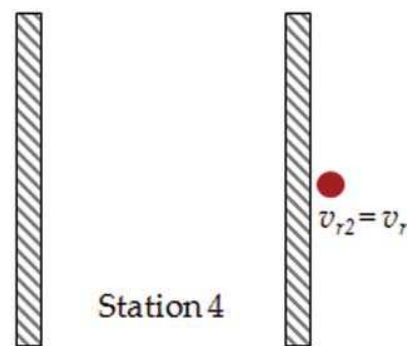

Back face-sheet (2)

FIG. 9. Application steps of the model to the spaced plate.

against penetration by the projectile. The secondary yarns have elastic deformation during the impact, are inside of the cone formed, and are not impacted directly by the projectile.

The model requires knowledge of the mechanical properties of the laminate (Young modulus, mechanical strength, ultimate strain, critical dynamic strain-energyrelease rate in mode II), the density and thickness of the plate, as well as the projectile parameters (geometry, mass, velocity, and diameter).

Given the energy conservation between two instants in time, such as an initial instant $\left(t_{0}\right)$ and a generic instant $\left(t_{\mathrm{i}}\right)$ :

$$
E_{\mathrm{KC}_{0}}=E_{\mathrm{T}_{i}}
$$

where, $E_{\mathrm{KC}_{0}}$ is the impact kinetic energy, $E_{\mathrm{T}_{i}}$ is the total energy in time $\mathrm{i}$.

The energies can be defined as:

$$
\begin{gathered}
E_{\mathrm{KC}_{0}}=\frac{1}{2} \cdot m \cdot v_{0}^{2} \\
E_{\mathrm{T}_{i}=E_{\mathrm{KC}_{i}}+E_{\mathrm{ED}_{i}}+} E_{\mathrm{FP}_{i}}=E_{\mathrm{KC}_{i}}+E_{\left.\mathrm{ED}_{(i} 1\right)} \\
+\Delta E_{\left.\mathrm{ED}_{(i, i} 1\right)}+E_{\mathrm{FP}_{(i 1)}}+\Delta E_{\left.\mathrm{FP}_{(i, i} 1\right)}
\end{gathered}
$$

where, $m$ is the mass of the projectile, $v_{0}$ is the velocity of the impact

$$
E_{\mathrm{FP}_{i}}=E_{\mathrm{TF}_{i}}+E_{\mathrm{DL}_{i}}+E_{\mathrm{MC}_{i}}
$$

$\Delta E_{\mathrm{FP}_{(i, i-1)}}$ and $\Delta E_{\mathrm{ED}_{(i, i-1)}}$ are the absorbed energy in the time interval between $t_{\mathrm{i}-1}$ and $t_{\mathrm{i}}$.
The velocity of the projectile for each instant of time $\left(v_{\mathrm{i}}\right)$ is: $v_{i}=\sqrt{\frac{1 / 2 \cdot m \cdot v_{0}^{2}-E_{\mathrm{FP}_{(i 1)}}+\Delta E_{\left.\mathrm{FP}_{(i, i} 1\right)}+E_{\left.\mathrm{ED}_{(i, i} 1\right)}+\Delta E_{\left.\mathrm{ED}_{(i, i} 1\right)}}{1 / 2\left(m+M_{C_{1}}\right)}}$

where, $M_{\mathrm{C}_{i}}$ is the mass of the cone formed on the back side of the plate

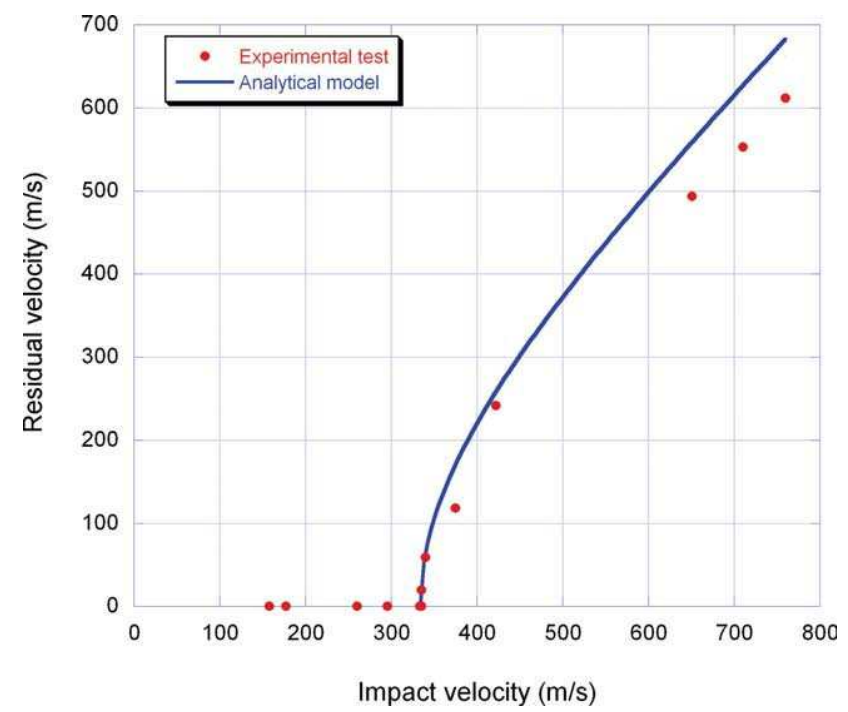

FIG. 10. Residual velocity vs. impact velocity of spaced plate. 
TABLE 2. Ballistic limit of spaced plate.

\begin{tabular}{lcc}
\hline Spaced plate & Experimental & Analytical \\
\hline Ballistic limit $(\mathrm{m} / \mathrm{s})$ & 335 & 333 \\
\hline
\end{tabular}

In this work, the model is applied to the spaced plate twice (Fig. 9), i.e. once for the front face-sheet, and another time for the back face-sheet. On the front facesheet the residual velocity (Station 2) was calculated and this velocity was used as the impact velocity in the back face-sheet (Station 3) and the residual velocity calculated in this face-sheet was identified as the residual velocity of the spaced plate (Station 4).

Figure 10 show the residual velocity calculated by the model and the experimental data, a good agreement with the experimental data was found. The differences between the experimental and analytical ballistic limit were negligible, and therefore it was possible to apply the model to estimate the ballistic limit and residual velocity of the spaced plate, which has a ballistic limit similar to the sandwich structure, as shown in the Figure 4 and Table 1. When the analytical model was applied the differences were $0.6 \%$, as shown in Table 2 .

The Figure 11 showed the percentage of absorbed energy regarding to the impact energy, determined with the analytical model on spaced plate, front-face sheet and back face-sheet. The percentage of energy absorbed by each face-sheet is similar for velocities higher than the ballistic limit. Below this velocity, the behavior of each face-sheet is different. In the back face-sheet the absorbed energy increases with the impact velocity and in the front face-sheet decreases. The percentage of absorbed energy

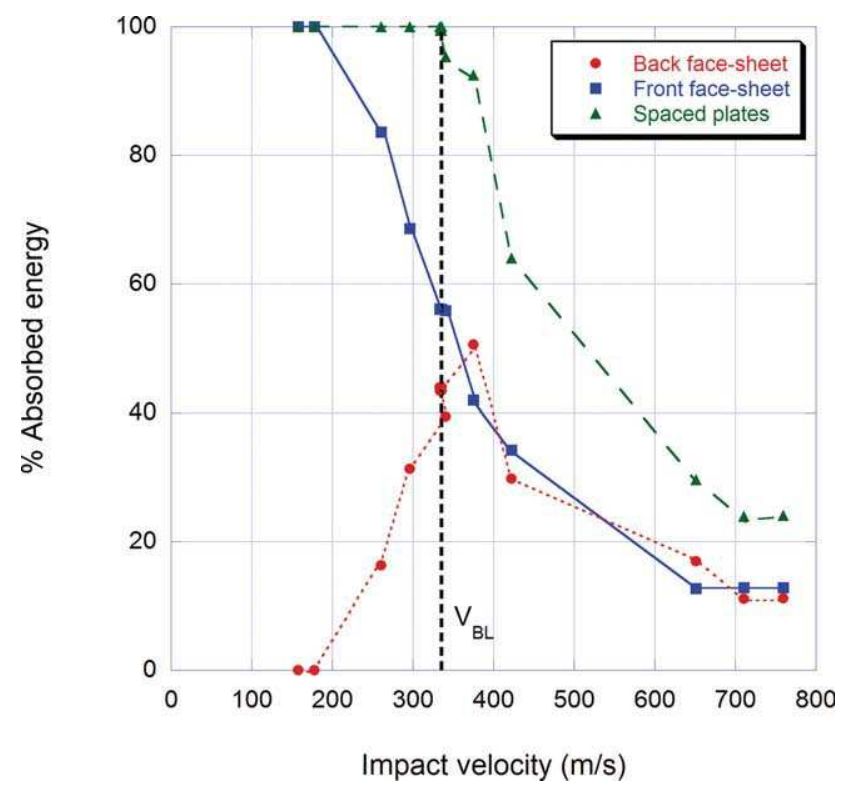

FIG. 11. Energy absorbed vs. impact velocity of spaced plates. by front face-sheet reduces with impact velocity because it is perforated in the range considered. However, in the back face-sheet the percentage increases with impact velocity because this face-sheet stopped the projectile and absorbed its kinetic energy. When both face-sheets are perforated the percentage of absorbed energy decreases when the impact velocity increases.

\section{CONCLUSIONS}

Composite structures subjected to high-velocity impacts were studied. Two kinds of structures were selected: sandwich structures and spaced plates of the same material and thickness. The ballistic limit was experimentally estimated for both structures, and the different between them was found to be $2.6 \%$. The damage generated by the impact was different, reducing the damage area in the front face-sheet of the space plate compared with the sandwich structure and increasing it in the back face. These differences could be explained by the propagation of elastic waves, which is controlled by the difference in the properties (density and Young modulus) between foam and air.

Additionally, the ballistic limit and absorbed energy in the spaced plates was predicted by an analytical model. The results agreed well with the experimental values.

\section{REFERENCES}

1. V. Rizov and A. Mladensky, Polym. Polym. Compos., 16, 4, (2008).

2. V. Koissin, V. Skvortsov, S. Krahmalev, and A. Shilpsha, Compos. Struct., 63, 34 (2004).

3. T. Gómez del Río, E. Barbero, R. Zaera, and C. Navarro, Compos. Sci. Technol., 65, 1, (2005).

4. W.J. Cantwell and J. Morton, Composites, 22 (1991).

5. S Abrate, Impact of Composite Structures, Cambribge Uni versity Press, UK (1998).

6. A.H. Sheikh, P.H. Bull, and J.A. Kepler, Compos. Sci. Tech nol., 69, 6 (2009).

7. D.W. Zhou and W.J. Stronge, Int. J. Impact. Eng., 35, 11 (2008).

8. V. Skvortsov, J. Kepler, and E. Bozhevolnaya, Int. J. Impact. Eng., 28, 7 (2003).

9. R. Velmurugan, M. Ganesh Babu, and N. K. Gupta, Int. J. Crashworthiness., 112 (2006).

10. C.C. Foo, L.K. Seah, and B.B. Chai, Compos. Struct., 85, 1 (2008).

11. M.S. Hoo Fatt and D. Sirivolu, Structures Under Shock and Impact $X, \mathrm{~N}$. JONES, University of Liverpool, UK and C.A. BREBBIA (2008).

12. M.S. Hoo and K.S. Park, Compos. Part A Appls., 31, 8 (2000).

13. MIL STD 662F Standard. $\mathrm{V}_{50}$ Ballistic test for armor. Department of Defense Test Method Standard (1997).

14. S. Ibekwe, P. Mensa, G. Li, and S. Pang, Compos. Struct., 79, 1 (2007). 
15. H. Kim, D. Welch, and K.T. Kedward, Compos. Part A Appls., 34, 1 (2003).

16. J. Christopherson, M. Mahinfalah, G. Nakhaie Jazar, and M. Rastgaar Aagaah, Compos Struct., 67, 3 (2007).

17. S.S. Morye, P.J. Hine, R.A. Duckett, D.J. Carr, and I.M. Ward, Compos. Sci. Technol., 60, 14 (2000).

18. N.K. Naik, P. Shrirao, and B.C.K. Reddy, Int. J. Impact. Eng., 32, 9 (2006).

19. J. López Puente, R. Zaera, and C. Navarro, Int. J. Solids. Struct., 44, 9 (2007).
20. S.K. García Castillo, S. Sánchez Sáez, J. López Puente, E. Barbero, and C. Navarro, Compos. Sci. Technol., 69, 6 (2009).

21. S.K. García Castillo, S. Sanchéz Sáez, E. Barbero, and C Navarro, J. Phys. IV., 134 (2006).

22. S.K. García Castillo, Análisis de laminados de materiales compuestos con precarga en su plano y sometidos a impacto, Ph.D Thesis, (2007), In spanish.

23. L.M. Nunes, S. Paciornik, and J.R.M. D'Almeida, Compos. Sci. Technol., 64, 78 (2004).

24. H. Kasano, JSME. Int. J. A Solid. M., 42, 2 (1999). 